\title{
GAIA Level 3 Neonatal Respiratory Tract Infection
}

National Cancer Institute

\section{Source}

National Cancer Institute. GAIA Level 3 Neonatal Respiratory Tract Infection. NCI

Thesaurus. Code C127993.

GAIA Level 3 Neonatal Respiratory Tract Infection is defined by the presence of two or more of the following criteria: a) Difficulty in breathing/tachypnea; b) Severe chest indrawing; c) Nasal flaring; d) Grunting; e) Wheeze; f) Stridor. 